\title{
Neurovascular lessons from a pair of identical twins with cerebral aneurysms
}

\author{
P Sharma, M J Brown
}

\begin{abstract}
A pair of hypertensive identical twins are reported, one of whom was non-compliant with her antihypertension medication, and after a subarachnoid haemorrhage was found to have multiple cerebral aneurysms. The other asymptomatic compliant twin was noted to have only a single small cerebral aneurysm. As both identical twins are likely to share the same cerebral vascular architecture it is suggested that good control of blood pressure contributed to the cerebral vascular integrity of the asymptomatic twin. This is the first report on the role of blood pressure in the formation of cerebral aneurysms and provides insights for an alternative approach to the controversial management of asymptomatic cerebral aneurysms. (Postgrad Med F 2001;77:197-198)
\end{abstract}

Keywords: cerebral aneurysm; identical twins; subarachnoid haemorrhage; hypertension

Subarachnoid haemorrhage $(\mathrm{SAH})$ is a significant cause of stroke and is associated with a case fatality rate of $40 \%-50 \%{ }^{1}$ The angiographic prevalence for unruptured intracranial aneurysms varies between $3.7 \%-6 \% .^{2} \mathrm{Al}-$ though smoking is an accepted risk factor, the role of hypertension has only recently been highlighted in a meta-analysis. ${ }^{1}$ These authors predicted that a reduction in blood pressure might result in a decreased incidence of SAH. ${ }^{1}$ Our case report demonstrates for the first time the importance of the role of hypertension in the formation and rupture of intracranial aneurysms.

Accepted 14 August 2000

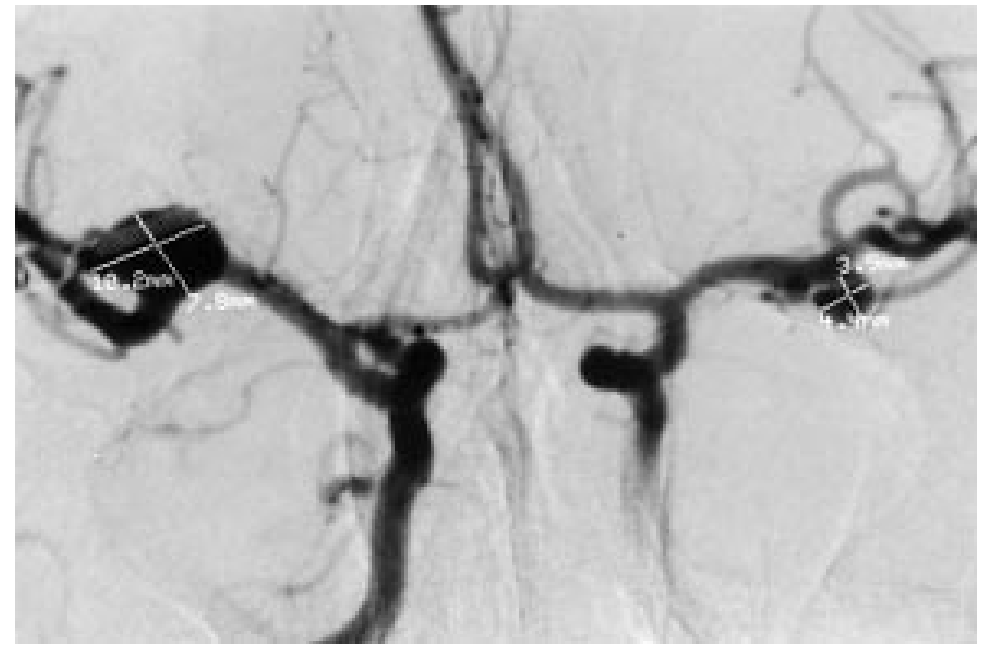

Figure 1 Multiple aneurysms in the poorly compliant hypertensive proband presenting with a subarachnoid haemorrhage.
Case report

A 44 year old white factory supervisor was referred for high blood pressure, apparently present since 29 years of age. Her mean clinic blood pressure was $177 / 99 \mathrm{~mm} \mathrm{Hg}$, random cholesterol $6.3 \mathrm{mmol} / \mathrm{l}$, normal urea and electrolytes, vanillylmandelic acid 18.0 $\mu \mathrm{mol} / 24$ hours (normal range 12-44), with a normal electrocardiogram and renal ultrasound. Her identical twin sister and two elder brothers were all hypertensives. Their mother suffered from two strokes at 77 and 80 years of age. Antihypertensive treatment with $\beta$-blockade and calcium channel blockade was titrated until a clinic reading of $124 / 74 \mathrm{~mm} \mathrm{Hg}$ was achieved on the combination of atenolol 50 $\mathrm{mg}$ and amlodipine $5 \mathrm{mg}$ once daily.

The patient missed follow up appointments, and 18 months later was admitted with a SAH. Computed tomography confirmed a large right frontotemporal intracerebral haematoma and a conventional angiogram revealed a giant right (and a smaller left) middle cerebral artery aneurysm as well as a right internal carotid aneurysm (fig 1). The right middle cerebral artery aneurysm was clipped. On recovery the patient admitted complete non-compliance over several months with her antihypertensive medication.

The asymptomatic identical twin sister who was known to be more compliant with her medication was reviewed and noted to have a blood pressure of 125/70 mm Hg on treatment. In view of the multiple aneurysms in her sister, magnetic resonance arteriography (MRA) was undertaken on this patient which suggested a small aneurysm in the right cavernous sinus. A formal cerebral angiogram was inconclusive but a repeat arteriogram one year later identified a $2.8 \mathrm{~mm}$ aneurysm on the right

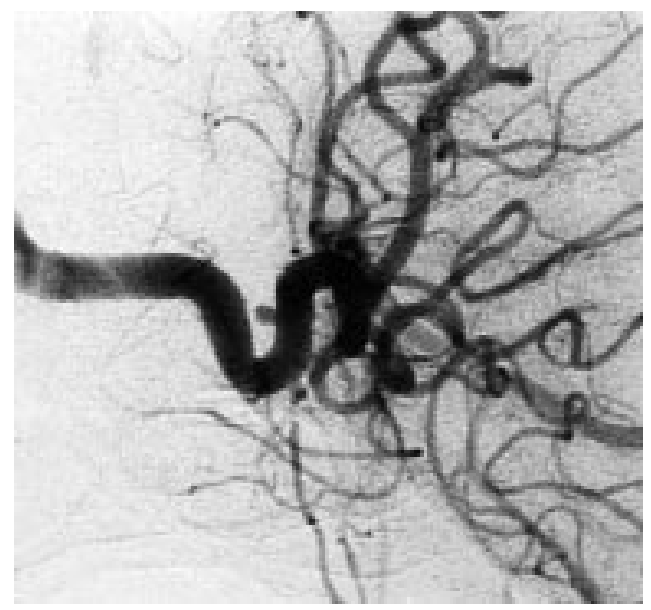

Figure 2 Analogous small solitary aneurysm in the compliant identical twin. 
carotid circulation (fig 2). Her blood pressure remains well controlled and the aneurysm is being followed up with MRA.

\section{Discussion}

Intracranial aneurysms are known to occur in families but the mode of inheritance is not clear. Although several rare disorders are associated with an increased risk for SAH and aneurysms, ${ }^{2}$ the most frequent relationship is among siblings (52\%) and it is suggested that this may be an obvious group of relatives in whom screening efforts should be targeted. ${ }^{2}$ The problem with screening, however, is that the natural history of such lesions is unknown but thought to relate to aneurysmal size and location. ${ }^{3}$ Moreover, there is debate about the choice of screening method and the suitability of available treatment options. ${ }^{2}$ Some investigators recommend screening when two or more members in the immediate family are affected, ${ }^{4}$ while others suggest mandatory screening in identical twins, ${ }^{5}$ although the benefits of such protocols have yet to be quantified. Whereas the quandary of screening was largely academic when the only mode of investigation, arteriography, carried risks not dissimilar to that of an aneurysm being present and subsequently rupturing, the situation has been changed by the widespread availability of non-invasive techniques of MRA.

Our case provides a unique opportunity to study the role of blood pressure in the development of cerebral aneurysms. The few previous reports of intracranial aneurysms in identical twins describe aneurysms, ${ }^{6}$ including rarely occurring multiple aneurysms, ${ }^{5}$ occurring at the same site. This suggests similarity between the architecture of the cerebral vessels of identical twins. Indeed, the fact that in our report both twins were hypertensive further supports the hypothesis that their vascular development was similar. That the treated normotensive compliant twin had only one visible aneurysm suggests that antihypertensive treatment was responsible for smaller and fewer aneurysms compared with the non-compliant twin.

We have no evidence to suggest that one class of antihypertensive drug has advantages over another but rather the level of blood pressure is more important. This leads us on to the next vexed question of what level of blood pressure ought to be aimed for? Clearly this question in relation to intracranial aneurysms has never been addressed but it seems sensible to aim for the lowest safest blood pressure tolerated. The recent HOT study (an international trial to determine such a level) suggests a level of 140/85 mm Hg without encountering difficulties. ${ }^{7}$ Obviously, the role of antihypertensives in asymptomatic normotensive relatives of patients with cerebral aneurysms needs more formal assessment but seems reasonable now to

\section{Learning points}

- Careful control of blood pressure may reduce both the development and incidence of SAH in at-risk individuals.

- High blood pressure is a risk factor for SAH but the greatest risk is a family history.

- Intracranial aneurysms can occur in families with the most frequent relationship being index patient to sibling.

- Small intracranial aneurysms $(<10 \mathrm{~mm})$ have a much lower chance of rupture compared with larger ones.

- Screening for asymptomatic aneurysms is controversial and should be undertaken on a patient-to-patient basis after a detailed family history.

recommend vigorous blood pressure reduction in any hypertensive sibling of patients with SAH.

There may be a genetic basis for ischaemic stroke $^{8}$ and more so with subarachnoid haemorrhages. ${ }^{9}$ The role of blood pressure in the formation of intracranial aneurysms has not previously been documented. The management of asymptomatic intracranial aneurysms is controversial as is screening relatives of patients with SAH. The present case suggests that rigorous control and monitoring of blood pressure in relatives of patients with symptomatic intracerebral aneurysms may be important in preventing their formation. As it is unclear whether relatives should be screened, or indeed what action should be taken if aneurysms are found, this case suggests an alternative and more palatable option.

PS is a BHF Clinician Scientist.

We are grateful to Drs R Higgins, G Elrington, and P Butler for their assistance with one of the twins.

1 Teunissen LL, Rinkel GJ, Algra A, et al. Risk factors for subarachnoid hemorrhage: a systematic review. Stroke 1996;27:544-9.

2 Wardlaw JM, White PM. The detection and management of unruptured intracranial aneurysms. Brain 2000;123:20521.

3 International Study of Unruptured Intracranial Aneurysms

Investigators. Unruptured intracranial aneurysms-risk of rupture and risks of surgical intervention. International rupture and risks of surgical intervention. International
Study of Unruptured Intracranial Aneurysms Investigators. Study of Unruptured Intracranial
$N$ Engl $F$ Med 1998;339:1725-33.

4 Schievink WI, Schaid DJ, Rogers HM, et al. On the inheritSchievink WI, Schaid DJ, Rogers HM, et al. On the inher
ance of intracranial aneurysms. Stroke 1994;25:2028-37.

5 Weil StM, Olivi A, Greiner AL, et al. Multiple intracranial aneurysms in identical twins. Acta Neurochirurg (Wien) 1988;95:121-5.

6 Puchner MJA, Lohmann F, Valdueza JM, et al. Monozygotic twins not identical with respect to the existence of intracranial aneurysms: a case report. Surg Neurol 1994;41:284-9.

7 Hansson L, Zanchetti A, Carruthers SG, et al. Effects of intensive blood-pressure lowering and low-dose aspirin in patients with hypertension: principal results of the Hypertension Optimal Treatment (HOT) randomised trial. Lancet 1998;351:1755-62.

8 Sharma P. Meta-analysis of ACE gene in ischaemic stroke. $\mathcal{F}$ Neurol Neurosurg Psychiatry 1998;64:227-30.

9 Kubota M, Yamaura Y, Ono J, et al. Is family history an independent risk factor for stroke? $\mathcal{f}$ Neurol Neurosurg Psychiatry 1997;62:66-70. 